\title{
Editorial: Hybrid quantum systems - new perspectives on quantum state control
}

\author{
S. Wimberger ${ }^{\mathrm{a}}$ \\ Institute for Theoretical Physics and Heidelberg Center for Quantum Dynamics, University of Heidelberg, \\ Philosophenweg 19, 69120 Heidelberg, Germany \\ Received 14 April 2011 \\ Published online 17 June 2011 - (C) EDP Sciences, Società Italiana di Fisica, Springer-Verlag 2011
}

\begin{abstract}
We give a brief sketch of the emerging field of quantum mechanical hybrid systems through a cross section of research papers on topics of current interest contained in this Topical Issue.
\end{abstract}

\section{What are hybrid quantum systems?}

The recent advance in technology and, in particular, in the experimental means of control of the quantum degrees of freedom on the atomic scale makes it possible nowadays to realise systems which consist of building blocks of different nature. Examples are nanomechanical systems coupled to a light field or nanoelectronic devices coupled to macroscopic leads which are nevertheless described quantum mechanically. Such systems are known as hybrid quantum systems. While major progress has been made in the area of nano- and optomechanical mechanical devices, see e.g. the review papers [1-3], more recently also other classes of systems have been thought of as possible building blocks of more complex devices. For the single ingredients of hybrid systems perfect tools have been developed over the last decades, mainly driven by the field of quantum and atom optics. For instance, charged atoms in ion traps, photonic fields on the quantum level, and, very importantly, ultracold atomic and molecular gases cannot only be produced and controlled with high precision, but are also used for applications, such as quantum information processing. The challenge lies in the combination of various systems and degrees of freedom of different nature and dimensions. One crucial point of interest is also the integration of systems, i.e. procedures to make them as small as possible, which would allow one the construction of merged systems on a individual substrate in a more or less standard manner.

Hybrid quantum systems are interesting interfaces of different quantum objects from microscopic to mesoscopic scales. Their novel properties can be expected to go much beyond the behaviour of their single constituents. This makes them perfect objects to investigate fundamental quantum effects occurring in the coupling of many degrees of freedom or of many particles/fields with each other. On the theoretical level many questions have still to be

\footnotetext{
a e-mail: s.wimberger@thphys.uni-heidelberg.de
}

answered since new concepts must be developed in order to reliably describe such complex quantum systems. Questions of decoherence and the influence of one part of the systems on another one or on the coupling to environments arise quite naturally in this context. Once such a true hybrid system is realised it will open the route to a variety of applications, ranging from high precision measurements to quantum state control and quantum information processing. Since many different physical realisations are thought of and currently being built and studied, the field of quantum hybrid systems is rather interdisciplinary. The exchange of ideas and methods of the different areas ranging from solid state physics to quantum chemistry lets us expect a very promising future. Figure 1 presents a schematic sketch of the interlinks of different components and interactions which have been realised already to a large extent in the laboratories. It also shows a selected list of possible applications and challenges to develop new theoretical and experimental techniques.

\section{This collection of papers}

The present Topical Issue consists of a selection of papers on various aspects and different areas involved in the study of hybrid systems. The focus lies on atomic physics and electronic or spin systems. This issue does not include contributions on the rapidly developing area of nano- and optomechanical systems. For state-of-the art information on these systems, the reader should consult the overviews given in references [1-3].

Superconducting devices have very interesting properties which can be used to engineer transport (see the paper by Soller and Komnik) and for experimental control (see the contributions by Bushev et al. and Cano et al.). A very fundamental application of the coupling of quite different degrees of freedom is the scattering of neutral atoms from surfaces and nanostructures which is addressed in 


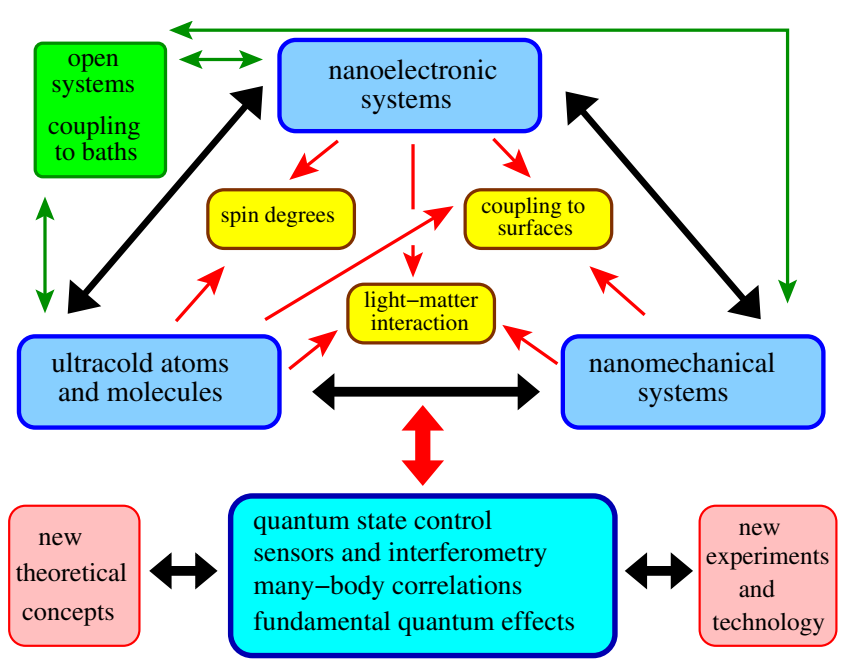

Fig. 1. (Color online) Schematic overview of various branches of physics which are interconnected in the study of quantum hybrid systems. The goals are stated below: the development of new theories and experiments/applications in which novel coupling mechanisms play a fundamental role.

the following papers by Jeske et al. and Fink et al. respectively. Transport in spin chains with focus on entanglement and control on the one hand, or as effective models for interband oscillations in tilted periodic potentials on the other hand is the topic of the contributions of Heule et al. and Plötz et al. Bose-Einstein condensates interacting with special forms of environments are then discussed by Hunn et al. and Trimborn et al. The last more fundamental paper by Hantschel and Fine discusses equilibration in abstract Hilbert spaces under certain constraints where unconventional results are expected.

The articles in this Topical Issue can of course not cover all aspects of the field of hybrid quantum systems, but they do provide a cross section of current research activities. We hope the articles will inspire the readers to discover new links between the various areas of research.

\section{Perspectives for future research}

I am sure that even during the compilation of this issue new research directions have been sprouting in the quest for hybrid quantum systems in theory and in experimental work. Other paradigms with high potential are, for instance, ultracold Rydberg atoms, which are by their pure geometrical size quasi macroscopic objects controlled by optical and magnetic potentials [4-6], or electronic spins coupled to microwave cavities [7].

During the past year there have been at least two scientific meetings which exclusively tried to bring together researchers of most of the fields participating in the quest for the realisation of novel states of quantum matter: one conference in Heidelberg in May 2010 entitled Hybrid Quantum Systems - New Perspectives on Quantum State Control ${ }^{1}$ and an intersectional symposium in the framework of the Spring Meeting of the German Physical Society in Dresden in March 2011 on Hybrid Quantum Systems - Interfacing Atoms, Solids and Light ${ }^{2}$. And more of such events are on the calendar in the near future.

Comparing the contributing papers makes it clear that the communities participating in the study towards working hybrid quantum systems will still have to learn from each other. My hope is that this Topical Issue will help to merge the jargons of the different communities and be a starting point for future research at the many frontiers of this fascinating field.

I am very grateful to my partners on the funding side, the Hengstberger Foundation for the Klaus-Georg and Sigrid Hengstberger Prize and the Heidelberg Graduate School of Fundamental Physics (DFG grant number GSC 129/1), and to the editorial board of the European Physical Journal D which made possible this Topical Issue. I acknowledge the help and the criticism broad forward by many referees for the papers of this issue, and my very special thanks goes to Ms Solange Guéhot for her professional and excellent assistance, which kept my own effort to a minimum.

\section{References}

1. T.J. Kippenberg, K.J. Vahala, Science 321, 1172 (2008)

2. F. Marquardt, S.M. Girvin, Physics 2, 40 (2009)

3. M. Aspelmeyer, Nature 464, 685 (2010)

4. T. Pohl, H.R. Sadeghpour, P. Schmelcher, Phys. Rep. 484, 181 (2009)

5. M. Weidemüller, Nature Phys. 5, 91 (2009)

6. M. Saffman, T.G. Walker, K. Mølmer, Rev. Mod. Phys. 82, 2313 (2010)

7. T. Duty, Physics 3, 80 (2010)

\footnotetext{
1 See http://www.thphys.uni-heidelberg.de/ ${ }^{\text {wimberge/ }}$ Hybrid.html

${ }^{2}$ See http://www.dpg-verhandlungen.de/2011/dresden/ syhq.html
} 